Supporting information 


\title{
Rapidly Self-deactivating and Injectable Succinyl Ester-based Bioadhesives for
}

\section{Post-operative Anti-adhesion}

\author{
Pengxu Wang, ${ }^{\dagger}$ Yuan Zhu, ${ }^{,},{ }^{\S}$ Luyao Feng, ${ }^{\dagger}$ Yinghui Wang, "Yazhong Bu* ${ }^{\dagger}$ \\ ${ }^{\dagger}$ Institute of Medical Engineering, Department of Biophysics, School of Basic Medical \\ Sciences, Health Science Center, Xi'an Jiaotong University, Xi'an 710061, China \\ ${ }^{\ddagger}$ Department of Reproductive Health, The Affiliated Maternal and Child Healthcare \\ Hospital of Nanchang University, Jiangxi 330006, China \\ ${ }^{\S}$ Department of Reproductive Health, Jiangxi Provincial Maternal and Child Health \\ Hospital, Nanchang, Jiangxi 330006, China \\ "College of Science, Chang'an University, Xi'an 710064, China \\ Pengxu Wang and Yuan Zhu contributed equally to this work. \\ *Corresponding author: Yazhong $\mathrm{Bu}$ \\ Email address: yazhongbu@xjtu.edu.cn
}




\section{Experimental section}

\section{Synthesis and Identification of PEG-NHS ester}

Polyethylene glycol (PEG) (Meryer) with molecular weight 8000 was used to generate the PEG grafted succinimide ester (PEG-NHS ester). Briefly, PEG, 4-dimethylaminopyridine (DMAP) (Aladdin, Shanghai), and succinic anhydride (Shaoyuan, Shanghai) (or glutaric anhydride) (RHAWN) with an equivalent ratio (1 eq: 2 eq: 5 eq) were added into a round bottom flask of $250 \mathrm{~mL}$. Then, about $100 \mathrm{~mL}$ anhydrous dichloromethane was poured into it, and the mixtures were reacted 10-12 $\mathrm{h}$ at $37{ }^{\circ} \mathrm{C}$. The reacted mixture was cleaned and centrifuged three times by $5 \%(\mathrm{w} / \mathrm{w})$ $\mathrm{NaCl}$ solution. Magnesium sulfate anhydrous was used to remove residual water. After that, the obtained mixture was treated by precipitation of petroleum ether, roto evaporation, and vacuum dry. Carboxyl terminated PEG was obtained. Then, carboxyl terminated PEG, EDC (Meryer), and NHS (Meryer) (equivalent ratio 1 eq: 2 eq: 5 eq) were added into the round bottom flask. The above post-process procedures were repeated to synthesize PEG-NHS ester.

\section{Preparation of SEgel and GEgel bioadhesive}

The PEG-SS or PEG-SG (pH=4.0) and gelatin (bloom 300, Sigma-Aldrich) $(\mathrm{pH}=9.0)$ were dissolved into phosphate solution at $20 \%$ and $25 \%(\mathrm{w} / \mathrm{w})$, respectively. Before injection, gelatin solutions were heated to $45^{\circ} \mathrm{C}$ to melt it and then added into one of the tubes of double syringes, and the PEG-SS or PEG-SG solutions were added into another tube. In addition, phosphate buffer $(\mathrm{pH}=7.0), 0.1 \mathrm{M} \mathrm{NaHCO}_{3}$ solution 
$(\mathrm{pH}=8.9)$ and phosphate buffer $(\mathrm{pH}=9.0)$ solution were used to test the gelation ability.

\section{Quantification of primary amine content}

The primary amine content of the gelatin solution was quantified by ninhydrin assay ${ }^{[1]}$

(ACMEC, Shanghai). $10 \mathrm{M} \mathrm{NaOH}$ (Guoyao), 50\% (vol/vol) 2-proponal (ACMEC, Shanghai), $0.5 \mathrm{mg} / \mathrm{mL}$ glycine stock solution (ACMEC. Shanghai), 4\% (wt/vol) ninhydrin solution (this solution needs to be wrapped by aluminum foil to avoid light), $200 \mathrm{mM}$ of citric acid (ACMEC, Shanghai) in $0.16 \%(\mathrm{wt} / \mathrm{vol})$ tin II chloride (ACMEC, Shanghai), were prepared. $100 \mu \mathrm{L}$ of $25 \%$ gelatin solution was diluted to $10 \mathrm{~mL}$ to detect the primary imine content. Briefly, $1 \mathrm{~mL}$ sample was added into $5 \mathrm{~mL}$ centrifuge tube, and $1 \mathrm{~mL}$ mixture (ninhydrin and tin II chloride at 1:1) was added to react $5 \mathrm{~min}$ at $100{ }^{\circ} \mathrm{C}$. Then, the reacted solution was cooled for $15 \mathrm{~min}$ at room temperature. $250 \mu \mathrm{L}$ of 2-propanol was added to stop the reaction, and $200 \mu \mathrm{L}$ liquid was added into 96 well to detect the absorbance at $570 \mathrm{~nm}$. The standard curve of the primary amine content was prepared in different $\mathrm{pH}$ solutions.

\section{Gelation time}

The gelation time was determined by the vial tilting method. The bioadhesives were prepared in sample vials. The time at which there was no flow upon inverting the vial was regarded as the gelation time.

\section{Comparison of Physical Characterization}


In vitro swelling analysis:

SEgel and GEgel adhesives were injected into a culture dish to gelatinize forming a disc with a radius of $75 \mathrm{~mm}$, and then were divided into four slices. The slices were placed into a centrifuge tube of $15 \mathrm{~mL}$ and phosphate buffer saline of $3 \mathrm{~mL}$ was added. The centrifuge tube was placed in a rotational table (THZ-100, Shanghai Yiheng scientific instruments Co., Ltd) and the speed was set as $120 \mathrm{rpm}$, and the temperature was $37^{\circ} \mathrm{C}$. The adhesives were weighted at fixed time $(12 \mathrm{~h}, 24 \mathrm{~h}, 36 \mathrm{~h}, 48 \mathrm{~h})$. The swelling ratio (SR) was calculated according to the equation: $S R=\left(\mathrm{W}_{2}-\mathrm{W}_{1}\right) \times 100 \%$. $\mathrm{W}_{2}$ represents the weight at a fixed time. $\mathrm{W}_{1}$ represents the initial weight.

In vitro degradation test:

SEgel and GEgel adhesives were injected into a culture dish to gelatinize forming a disc with a radius of $75 \mathrm{~mm}$, and then were divided into four slices. The slices were placed into a centrifuge tube of $15 \mathrm{~mL}$ and phosphate buffer saline of $3 \mathrm{~mL}$ was added. The centrifuge tube was placed in a rotational table (THZ-100, Shanghai Yiheng scientific instruments Co., Ltd) and the speed was set as $120 \mathrm{rpm}$, and the temperature was $37^{\circ} \mathrm{C}$. The remaining weight of adhesives was recorded at fixed time (1day, 2 days, 3 days, 4 days, 5 days, 6 days, 8 days).

\section{In vivo degradation test by subcutaneous implantation}

3D printer with polylactic acid ink was used to print the ring mold with an external diameter of $6 \mathrm{~mm}$, an internal diameter of $5 \mathrm{~mm}$, and a height of $2 \mathrm{~mm}$. SEgel and 
GEgel adhesives were injected into the mold, and the cured adhesives had a similar weight with 40-50 mg. After that, SEgel and GAEel adhesives disk was sterilized for 30 minutes under UV light. Female Sprague Dawley rats with a bodyweight of 140-160 g were used. Two subcutaneous pockets were created by the incision of $1 \mathrm{~cm}$ on the dorsal skin $(5 \mathrm{~cm}$ from the connecting line of both ears, $1 \mathrm{~cm}$ from the midline of the dorsum). GEgel adhesives were implanted in the right dorsum, and SEgel adhesives were left $(n=6)$. The incised pockets were sutured by 3.0 non-absorbable sutures, and the rats were returned to their cages to recovery. At 4 and 10 days, respectively, the groups of rats were executed by cervical dislocation. The morphology of adhesives was recorded by a camera, and the adhesives with surrounding tissue were cut and treated to perform histopathological examination.

\section{Rheological behaviors of SEgel and GEgel bioadhesives}

SEgel and GEgel bioadhesives and gelatin were injected into the ring model respectively with a $3 \mathrm{~cm}$ diameter and cured to form a disk completely. All the hydrogels were incubated in the incubator at $37{ }^{\circ} \mathrm{C}$ for $30 \mathrm{~min}$ before testing. Frequency-sweep curves (angular frequency 100-0.1 rad/s, strain of $1 \%, 37^{\circ} \mathrm{C}$ ) and time-sweep curves (0-220 s, interval $10 \mathrm{~s}, 1 \mathrm{~Hz}$, strain of $\left.1 \%, 37{ }^{\circ} \mathrm{C}\right)$ were performed in a rheometer (Anton Paar, Shanghai). Gelatin alone was used as control.

Scanning electron microscope (SEM) and energy dispersive spectrometer (EDS) analysis 
SEgel and GEgel bioadhesives and gelatin were pre-frozen in $-80{ }^{\circ} \mathrm{C}$ and then were lyophilized. The adhesives disk was sprayed with gold and subjected to SEM-EDS analysis (Tianmei, China).

\section{$X$-ray photoelectron spectroscopy (XPS)}

XPS was carried out on a Thermo ESCALAB 250XI. Survey scans were run at a pass energy of $100 \mathrm{eV}$. High-resolution scans were run at $30 \mathrm{eV}$. Carbon $1 \mathrm{~s}=284.8 \mathrm{eV}$ is the standard for charge calibration.

Attenuated total reflection Fourier transform infrared spectroscopy (ATR-FTIR)

ATR-FTIR analysis was carried out on a Thermo Scientific Nicolet iS5. The wavenumber range is $4000 \sim 400 \mathrm{~cm}^{-1}$.

\section{Molecular dynamics simulation}

The electronic geometries of the reactant $(\mathrm{R})$ and product $(\mathrm{P})$ were optimized using the B3LYP density functional with the standard basis sets of $6-31++\mathrm{G}(\mathrm{d}, \mathrm{p})$. The nature of stationary points as either local minima ( $\mathrm{R}$ and $\mathrm{P}$ ) or first-order saddle points (TS) was confirmed by the analysis for harmonic frequency, which was performed at the same level of theory. To obtain more accurate energies, single point calculations for reactants and products were performed with the B3LYP level of theory and standard basis sets of $6-311++\mathrm{G}(\mathrm{d}, \mathrm{p})$ using the above geometries, where bulk solvation effects were simulated by the explicit water molecules combined with 
integral equation formalism-polarized continuum model (IEF-PCM). Zero-point vibrational energy derived from the frequency calculations was extracted to obtain the relative free energy for all related species. All the calculations were performed using the Gaussian 16 program package.

\section{Adhesion strength Test}

Porcine skin was used as a medium to test the bonding strength since it resembles human tissue ${ }^{[2]}$. The porcine skin was purchased by local shopping and was cut into a rectangle shape $(2 \mathrm{~cm} \times 1 \mathrm{~cm})$. About $250 \mu \mathrm{L}$ of adhesives (SEgel and GEgel) was injected on the skin surface, and the pressing time was 1 minute. The bonding strength was tested in the form of lap-shear strength at room temperature by a tensile test machine with a force sensor of 50 N (CMT1103, Zhuhai Sansi Test Equipment Co., Ltd, China). The stringing rate was maintained at $10 \mathrm{~mm} / \mathrm{min}$ and each group at least repeated 3 samples ( $\mathrm{n} \geq 3$ ). The rupture force was recorded and converted into adhesion strength according to it is the function of the contact area.

\section{Self-deactivation test}

To evaluate the self-deactivating feature of adhesives, porcine skins were cut into a rectangle shape $(2 \mathrm{~cm} \times 1 \mathrm{~cm})$. The adhesives were injected on the prepared porcine skin by double syringe and $0,1,3,5,20$ and 50 minutes waited. And then, another porcine skin was pressed on the site of adhesives and the contacted area was about $1 \mathrm{~cm}^{2}$. The pressing time was maintained at 1 minute, and the incubating time was 
kept at $60 \mathrm{~min}$.

\section{In vitro cytotoxicity test:}

The cytotoxicity test including degradation products and maceration products of SEgel and GEgel bioadhesives were tested by CCK-8. The SEgel and GEgel adhesives were sterilized for 30 min under a UV atmosphere. For degradation product cytotoxicity (SEgel and GEgel), $1 \mathrm{~g}$ of adhesives was soaked in $\mathrm{NaOH}$ solution (1mL, $0.2 \mathrm{M}$ ) for $24 \mathrm{~h}$ and $0.2 \mathrm{M} \mathrm{HCl}$ was used to adjust the $\mathrm{pH}$ of maceration medium to 7.4. After that, the maceration medium was mixed with $8 \mathrm{~mL}$ of Dulbecco's modified Eagle medium-high glucose (DMEM-H, Solarbio). As to maceration product cytotoxicity (SEgel and GEgel), $1 \mathrm{~g}$ of adhesives was soaked in the $10 \mathrm{~mL}$ of DMEM-H to maintain $24 \mathrm{~h}$. The treated culture medium was filtered by a $0.22 \mu \mathrm{m}$ of poly(ether sulfones) filter (Sartotius). Before use, $1 \mathrm{~mL}$ of fetal bovine serum was added into the treated medium and then it was diluted into $10 \mathrm{mg} / \mathrm{mL}, 1 \mathrm{mg} / \mathrm{ml}$, and $0.1 \mathrm{mg} / \mathrm{mL}$ to test cytotoxicity. NIH $3 \mathrm{~T} 3$ mouse fibroblasts were plated in 96 -well plates with a density of $1 \times 10^{4}$ cells $/ 100 \mu \mathrm{L}$ at $37^{\circ} \mathrm{C}$ in $5 \% \mathrm{CO}_{2}$ for $24 \mathrm{~h}$. Then, supernate was removed and $100 \mu \mathrm{L}$ of various treated culture medium was added to culture 24 h. $10 \mu \mathrm{L}$ of CCK-8 was used to detect the cytotoxicity. The detective wavelength was $450 \mathrm{~nm}$ and the reference wavelength was $620 \mathrm{~nm}$ in a microplate reader (SuperMax 3100, Shanghai Shanpu Biotechnology Co., Ltd). The cell viability higher than $70 \%$ was recognized as non-cytotoxicity. Cell viability was calculated according to the equation: $\mathrm{CV}=\left[\left(\mathrm{W}_{\text {test }}-\mathrm{W}_{\text {blank }}\right) /\left(\mathrm{W}_{\text {control }}-\mathrm{W}_{\text {blank }}\right)\right] \times 100 \%$. 


\section{In vitro cell adhesion study:}

$50 \mathrm{mg}$ of gelatin was dissolved in $0.1 \mathrm{M} \mathrm{PB}$ solution with $\mathrm{PB}=9.0$ and heated at $45{ }^{\circ} \mathrm{C}$ to melt. SEgel and GEgel crosslinker $(40 \mathrm{mg} / \mathrm{mL})$ was dissolved in $0.1 \mathrm{M}$ PB solution with $\mathrm{pH}=4.0$. Gelatin and crosslinker were mixed and reacted for 10 minutes and then injected into 48 well plates. The plates were placed in a vacuum drying oven until the water evaporated to generate a thin film. The control group only had gelatin but not the crosslinker. 3T3 fibroblasts and RAW macrophage were digested from the culture dish and planted into the 48 -well plates to incubate for $2 \mathrm{~h}$ under $22{ }^{\circ} \mathrm{C}$ in a free FBS culture medium. The supernatant was discarded and washed three times by $\mathrm{pH}=7.4$ phosphate buffer to remove non-adhesion cells. $500 \mu \mathrm{L}$ of cell fixation fluid were added and incubated for 15 minutes. $\mathrm{pH}=7.4$ phosphate buffer was used to wash three times and $50 \mu 1$ DAPI was used to stain the cell nucleus. The cell count was carried out in a fluorescence microscope (Leica).

\section{Magnetic resonance imaging}

Magnetic resonance imaging (MRI) was conducted with a $1.5 \mathrm{~T}$ magnetic field intensity (Niumai, China). SD rats were under an air anesthesia system with a $1.5 \%$ isoflurane flow speed. Spin echo mode with T1-weight (20-400) and T2-weight (80-1500) sequence through axial imaging (Figure S9) were employed to determine the thickness variation of the implant at 1 day, 2 days, 3 days, 4 days, 5 days, 7 days, 10 days, respectively. 


\section{Cecum-sidewall adhesion model}

Female Sprague Dawley rats with a bodyweight of 140-160 g were used. The experiments were carried out in accordance with the Xi'an Jiaotong University Standing Committee on the Use of Animals in Research and Teaching (No.2021-213). All SD rats were anesthetized by isoflurane with a flow speed of $2 \%$ and $5 \%$ of airflow speed. Abdomen fur was shaved and $75 \%$ alcohol was used to disinfect. The abdomen skin was cut along the midline and the incision was about $3 \mathrm{~cm}$. The cecum was taken out using a toothbrush to rub the serosa to form a uniform bleeding point. The corresponding abdominal parietal membrane was rubbed by a scalpel. The wound of the experimental group was covered by the SEgel and GEgel adhesives about 400 $\mu \mathrm{L}$ and 3 minutes were waited for gelation. And then, the cecum was returned to the abdominal cavity, and the incision was sutured by a 3-0 suture. The wound was sterilized with iodine. The cecum with a rub but without adhesives was a control check. The adhesion score was evaluated by a blinded pathologist.

\section{Hepatic adhesion Model}

Female Sprague Dawley rats with a bodyweight of 140-160 g were used. The experiments were carried out in accordance with the Xi'an Jiaotong University Standing Committee on the Use of Animals in Research and Teaching. All SD rats were anesthetized by isoflurane with a flow speed of $2 \%$ and $5 \%$ of airflow speed. The chest fur was shaved and $75 \%$ alcohol was used to disinfect. $3 \mathrm{~cm}$ of incision was created and the distractor was used to further open the thoracic cavity. The left lateral 
lobe of the rat liver was taken out, and the underpart was turned over. A round tissue sampler with a diameter of $5 \mathrm{~mm}$ was used to fix position, and a cross wound was created by scalpel. After that, the thoracic cavity was closed and sutured by 3-0 suture. The wound was sterilized with iodine. The liver with a rub but without adhesives was a control check. The adhesion score was evaluated by a blinded pathologist.

\section{Histopathologic analysis}

In the fixed time, SD rats were euthanized and tissues were fixed with $4 \%$ paraformaldehyde (PFA)/PBS at $4{ }^{\circ} \mathrm{C}$ overnight, followed by gradient ethanol dehydration, paraffin embedding, pathological section and then treated for Hematoxylin and Eosin (H\&E) and Masson staining. The degree of inflammation was evaluated by a blinded histopathology expert.

\section{Statistical analysis}

For multiple comparisons, one-way ANOVA with a Duncan test was used. For nonparametric data, Mann-Whitney U was used. 


\section{Supporting Figures}

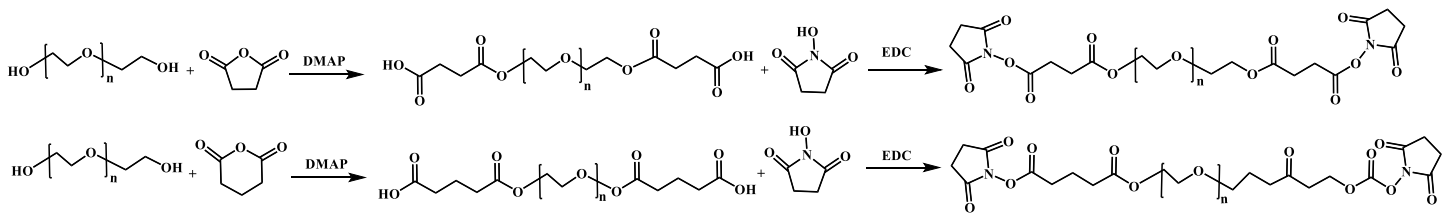

Figure S1. Synthetic pathway of PEG-SS and PEG-SG crosslinker

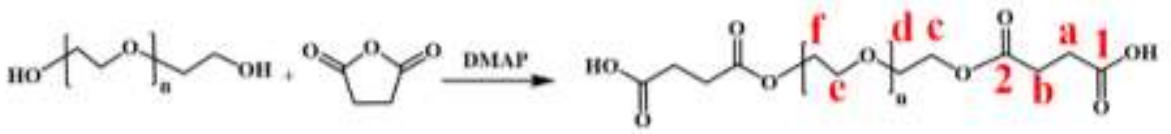

\section{a}
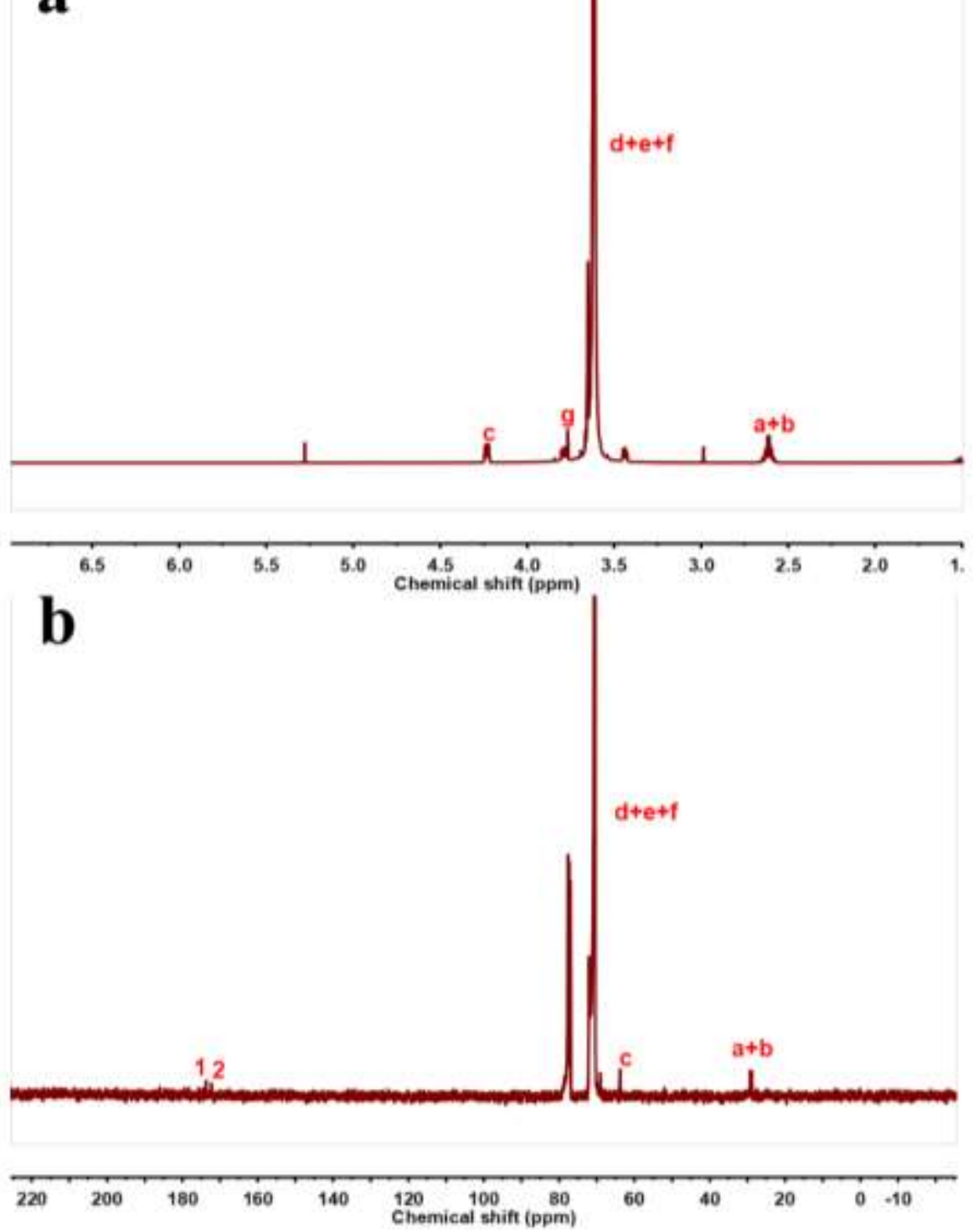

Figure S2. Poly(ethylene glycol) succinic acid was identified by ${ }^{1} \mathrm{H}$ NMR (a) and (b) 
${ }^{13} \mathrm{C}$ NMR spectra in $\mathrm{CDCl}_{3}$.
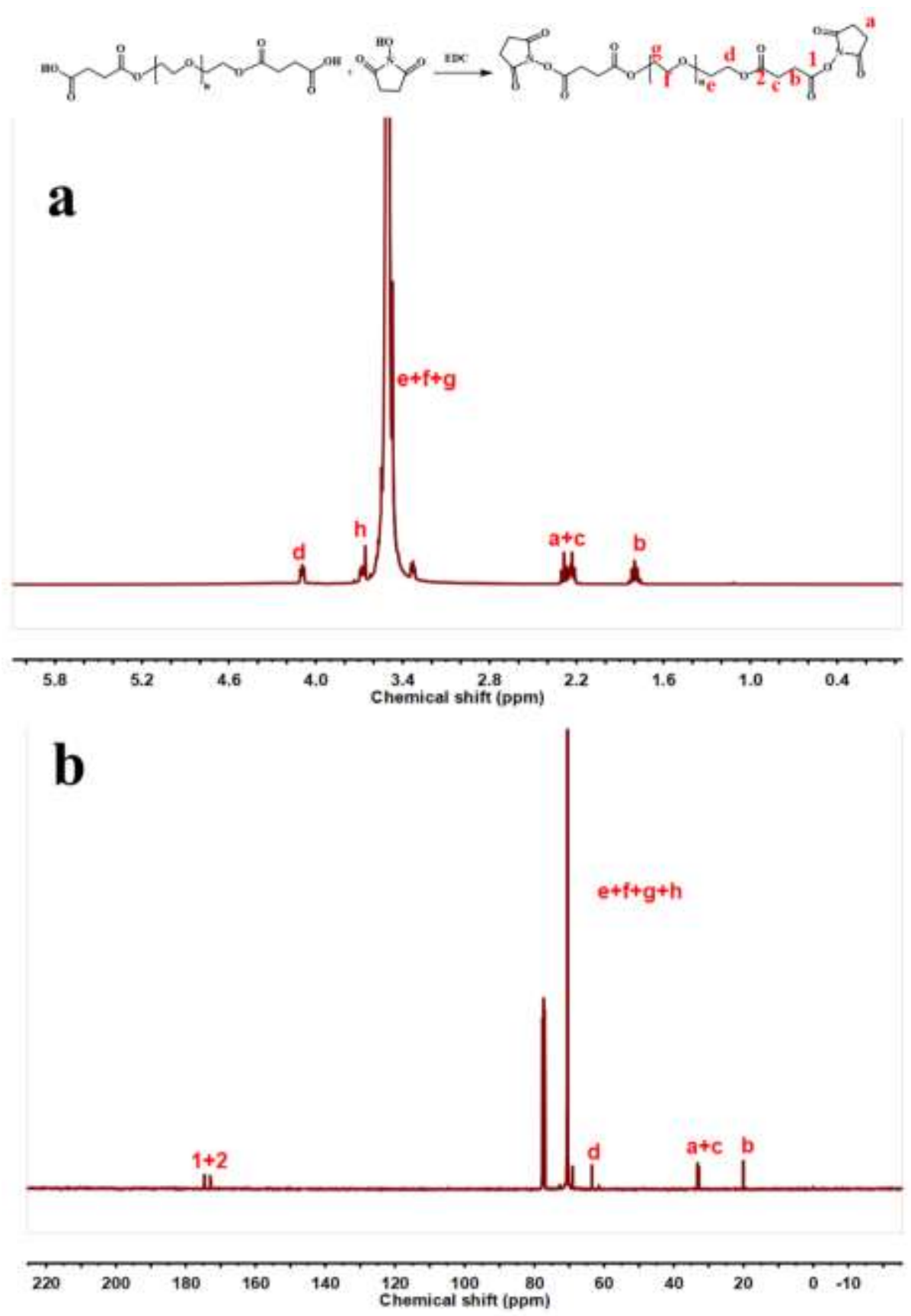

Figure S3. PEG-SS was identified by ${ }^{1} \mathrm{H}$ NMR (a) and (b) ${ }^{13} \mathrm{C}$ NMR spectra in $\mathrm{CDCl}_{3}$. 

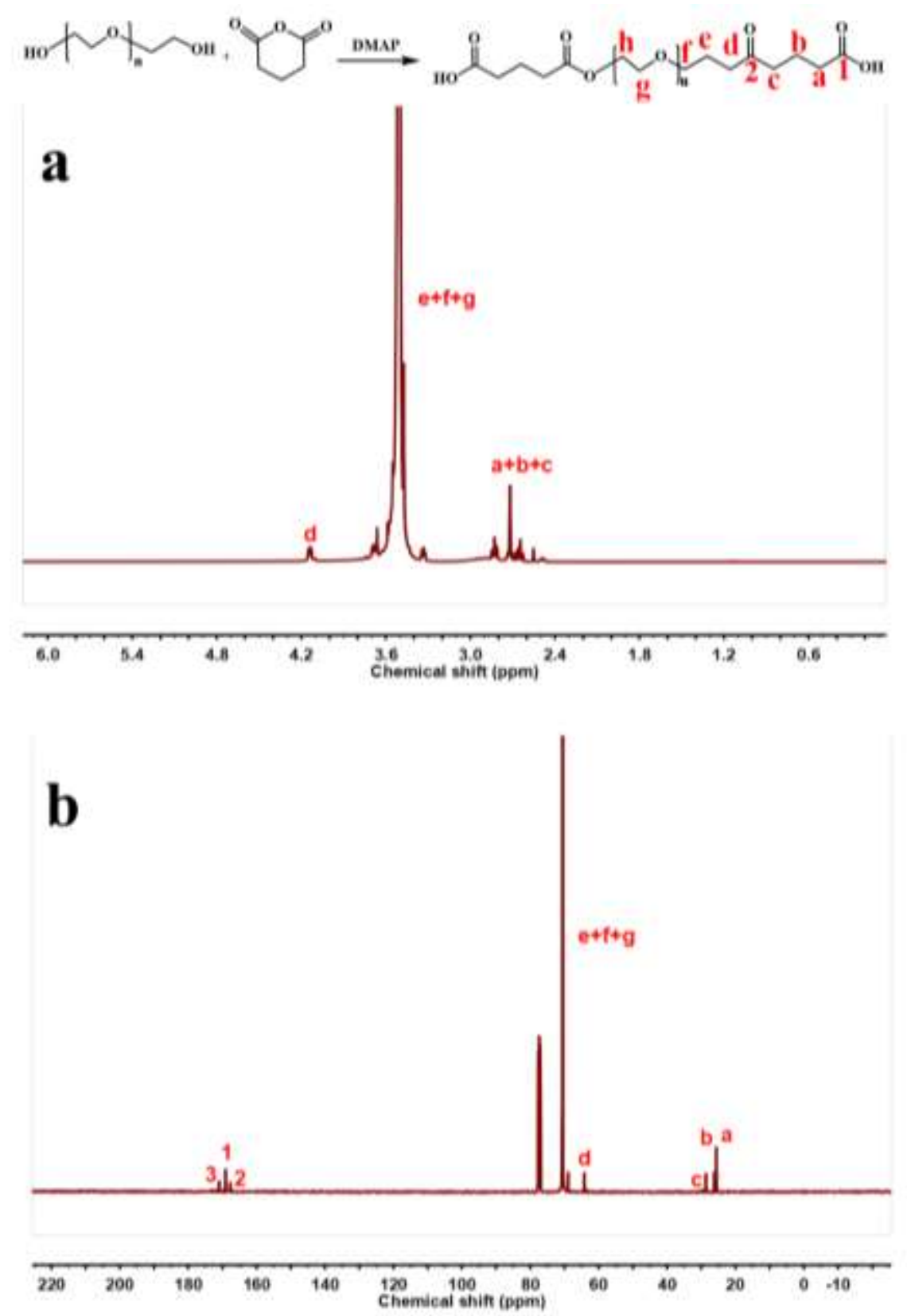

Figure S4. Poly(ethylene glycol) glutarate acid identified by ${ }^{1} \mathrm{H}$ NMR (a) and ${ }^{13} \mathrm{C}$ NMR (b) spectra in $\mathrm{CDCl}_{3}$. 

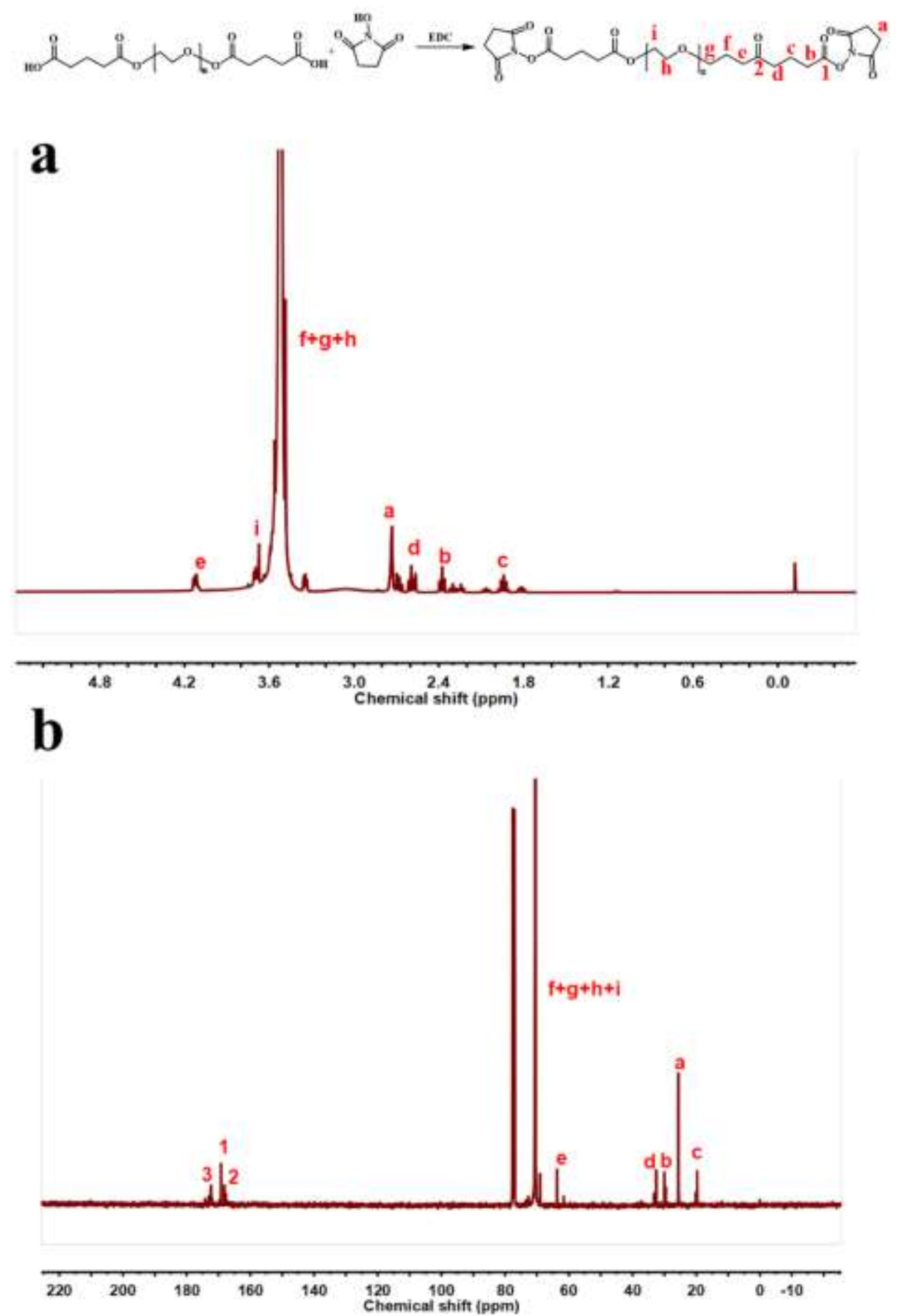

Figure S5. PEG-SG was identified by ${ }^{1} \mathrm{H}$ NMR (a) and (b) ${ }^{13} \mathrm{C}$ NMR spectra in $\mathrm{CDCl}_{3}$. 


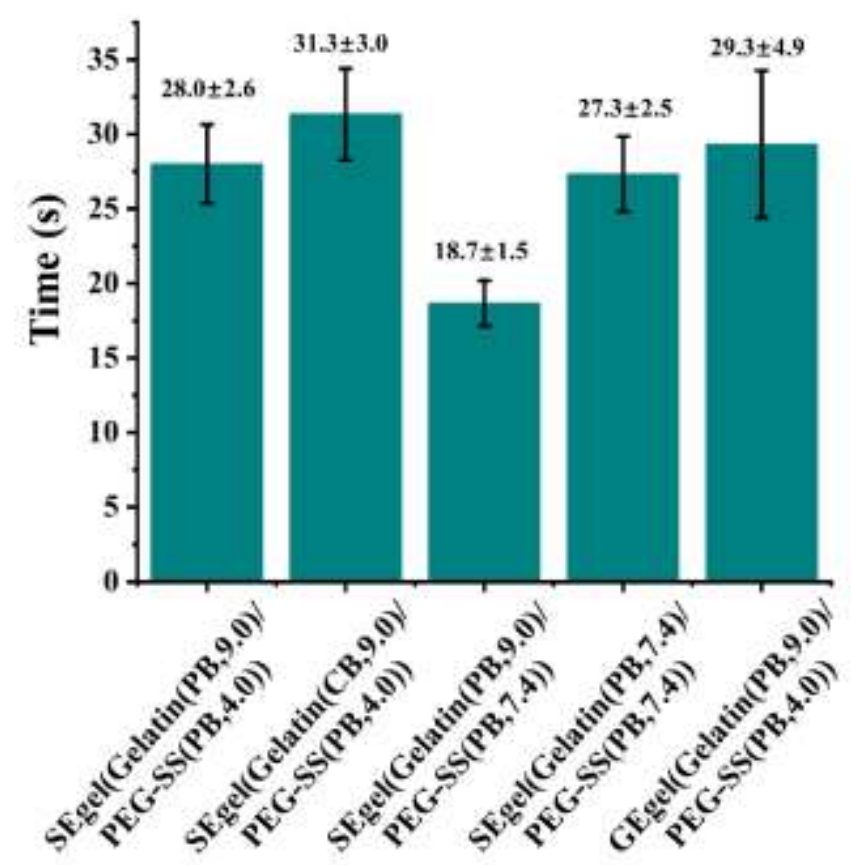

Figure S6. Gelation time of SEgel and GEgel using different buffer systems. (n=3)

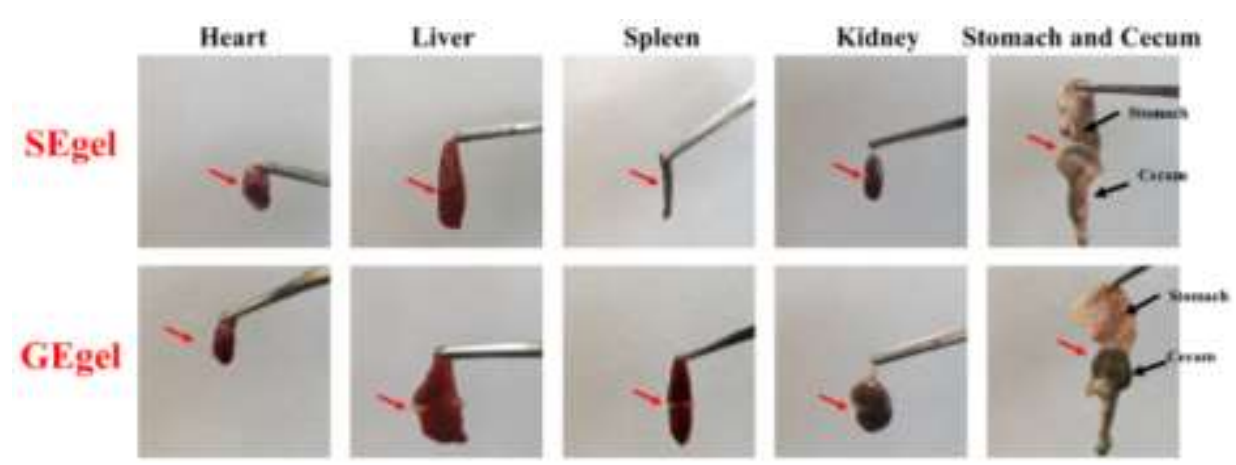

Figure S7. Pictures showing that SEgel and GEgel adhered cross-sectioned heart, liver, spleen, and kidney together. It also adhered stomach and cecum together. The red arrows indicate places for adhesion.

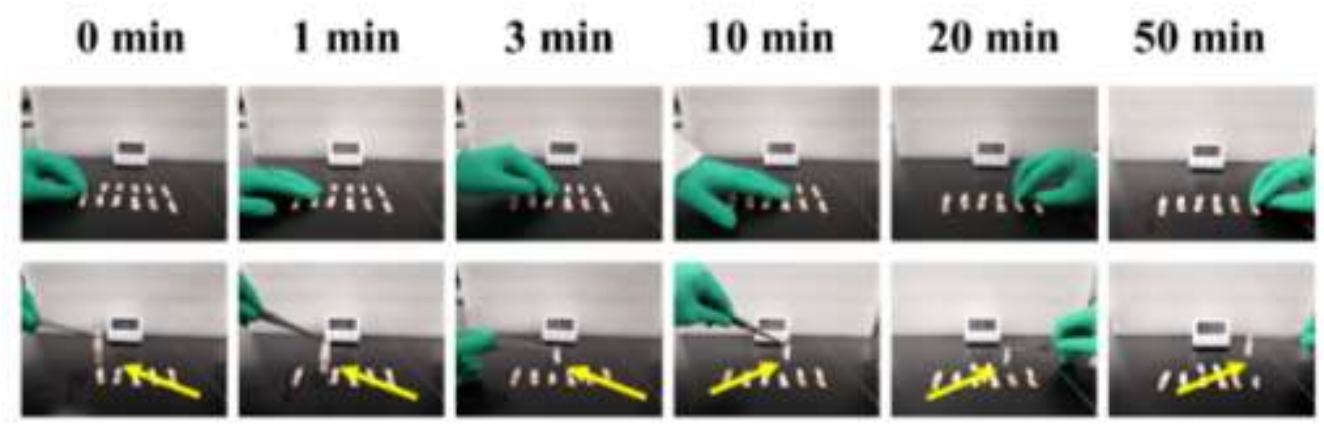


Figure S8. Self-deactivating verification of GEgel. The GEgel was injected on the porcine skin and after $0,1,3,10,20,50 \mathrm{~min}$, another skin was placed on the adhesives with a pressing time of 1 minute. After 60 minutes of incubation, the upper porcine skin was held up to see if the bonding was formed.
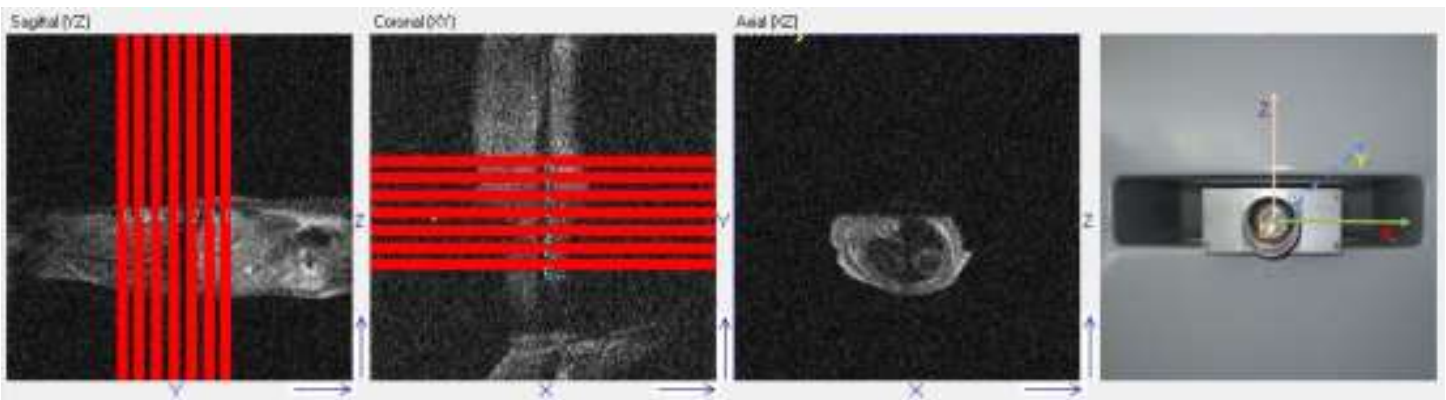

Figure S9. The sagittal, coronal and axial imaging of SD rats in a pre-scan model to localize the bioadhesives. The final scanning model was carried out in axial imaging (XZ axial).

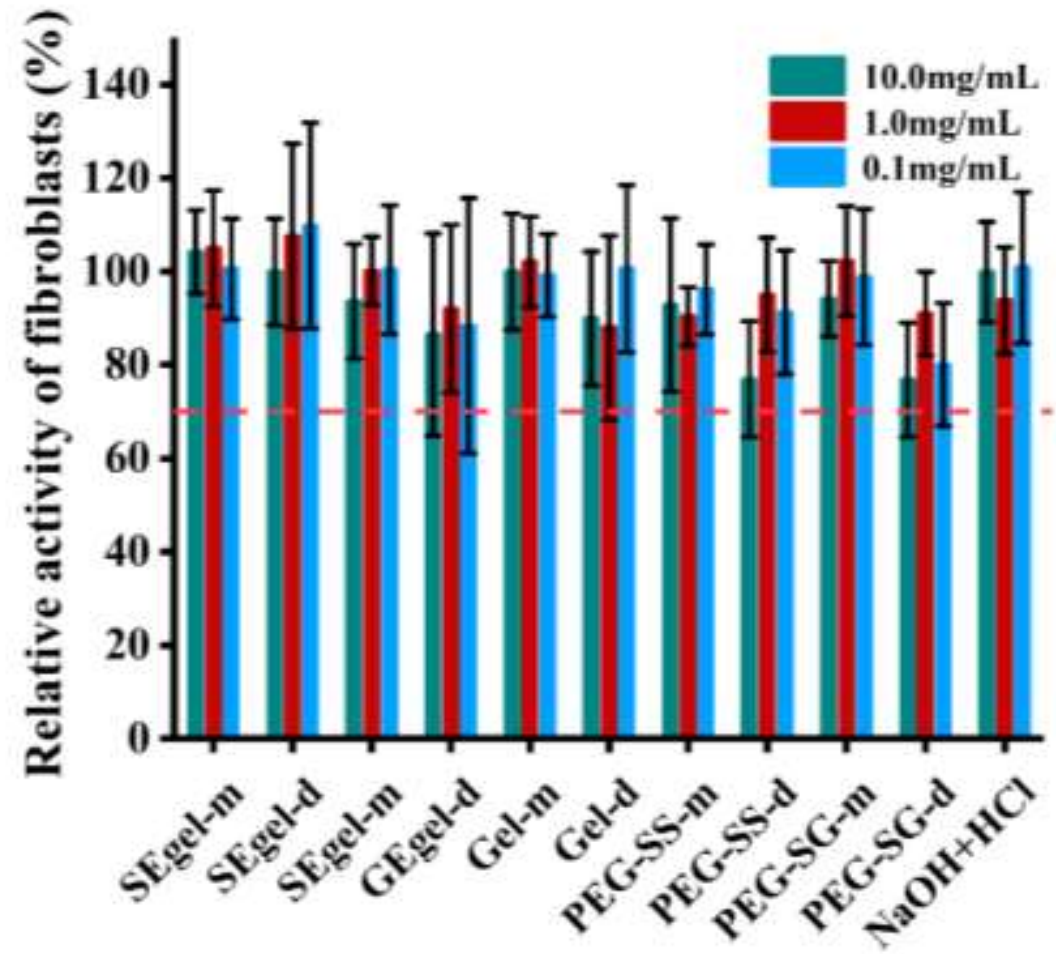

Figure S10. In vitro cytotoxicity in maceration and degradation products was compared by quantifying the relative viability of NIH $3 \mathrm{~T} 3$ fibroblasts $(n=6) . m$, maceration; d, degradation; Gel, gelatin; Relative activity $>70 \%$ was deemed as no-cytotoxicity. Data are reported as mean \pm SD and analyzed by a one-way ANOVA with a Duncan test. 


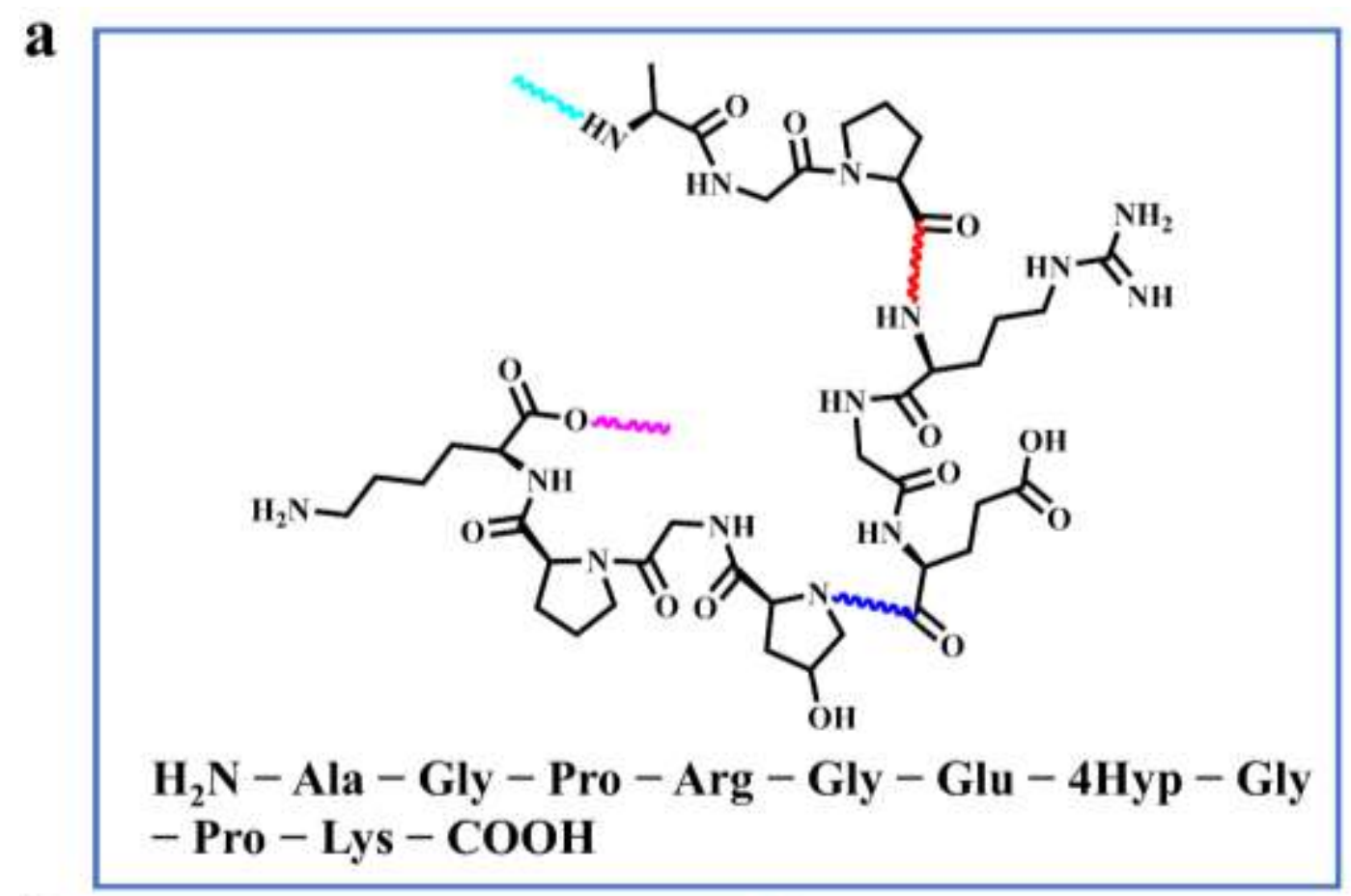

b
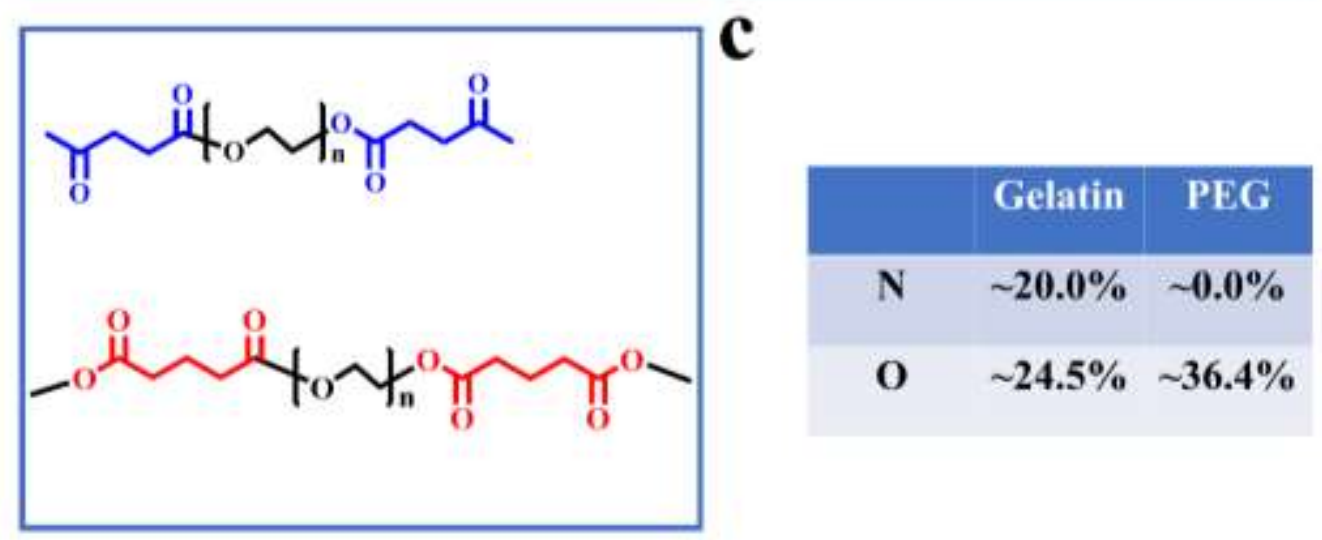

Figure S11. (a) The structure of simplified gelatin. (b) The structure of PEG-SS or PEG-SG after reacting with gelatin. (c) Mass fraction of $\mathrm{O}$ element and $\mathrm{N}$ element in gelatin and PEG. 

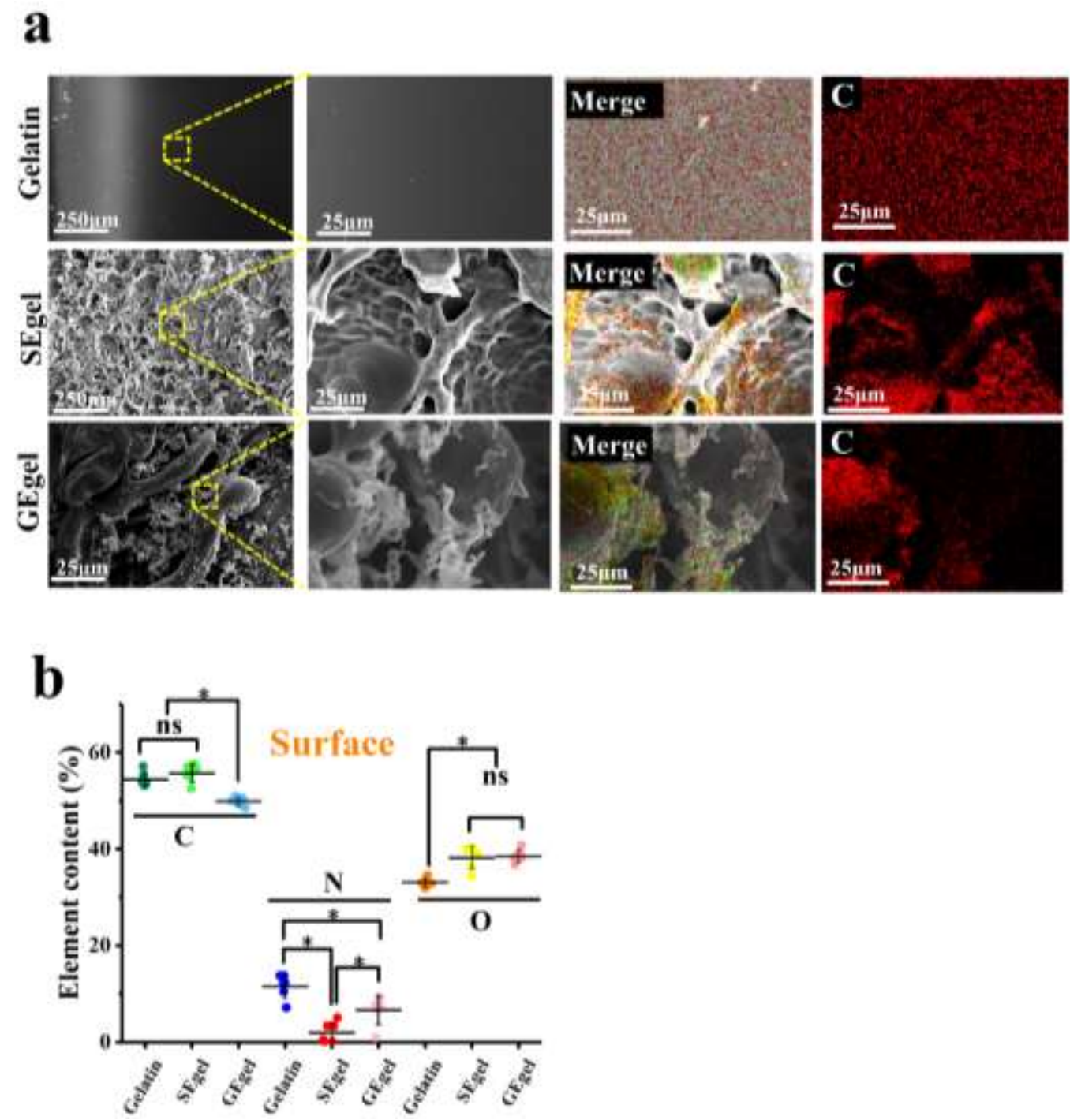

Figure S12. (a) Ultrastructure of surface, merge graph of $\mathrm{C}, \mathrm{N}$ and $\mathrm{O}$ element and distribution of C element of gelatin, SEgel and GEgel; (b) Element content analysis of gelatin, SEgel and GEgel. Data are reported as mean \pm SD and analyzed by a one-way ANOVA with a Duncan test. $* P<0.05$ between groups. 

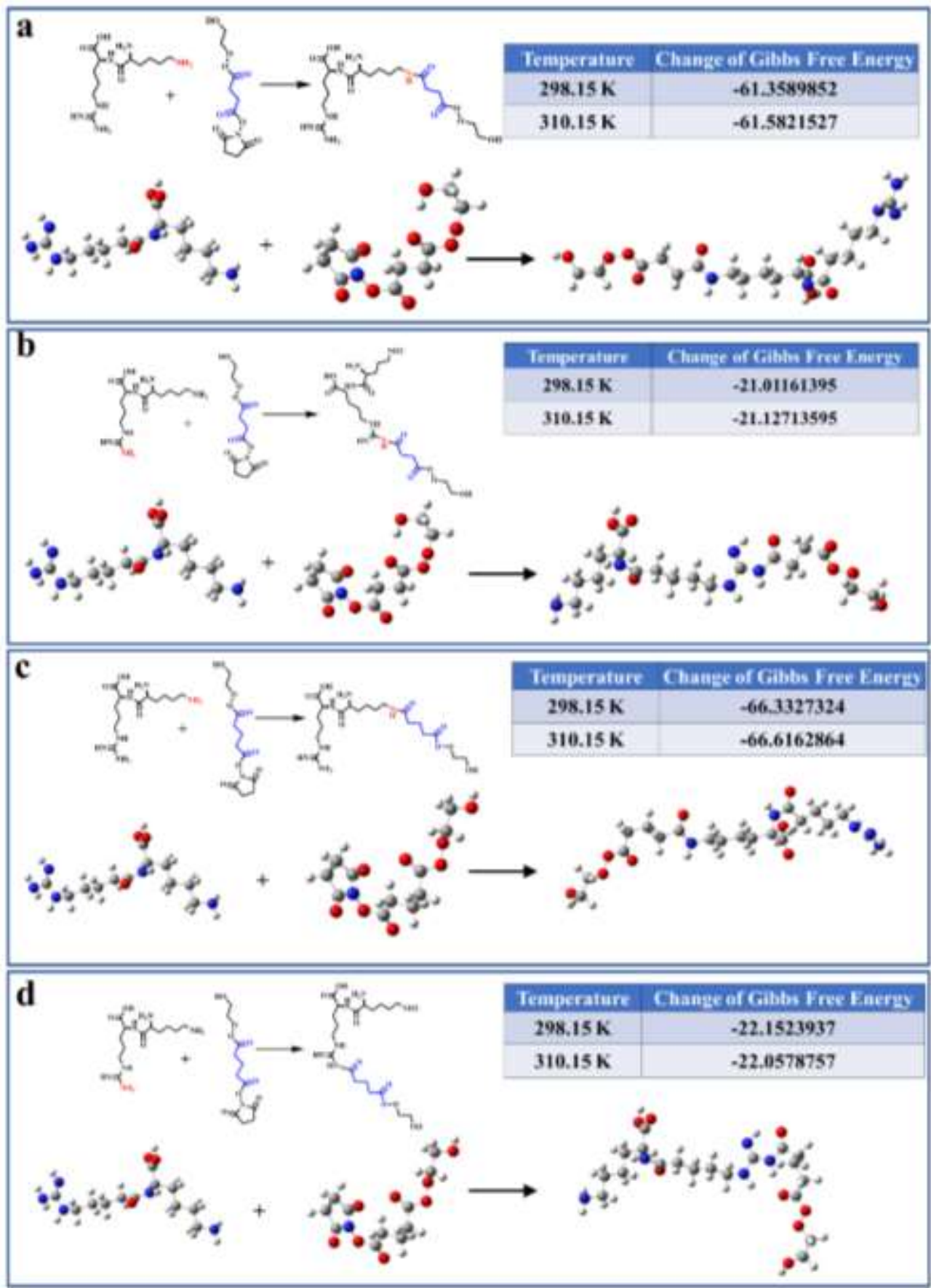

Figure S13. Simulation of reactivity of gelatin and PEG-SS or PEG-SG. (a) The reactivity of $-\mathrm{NH}_{2}$ of lysine from simplified gelatin with PEG-SS. (b) The reactivity of $-\mathrm{NH}_{2}$ of arginine from simplified gelatin with PEG-SS. (c) The reactivity of $-\mathrm{NH}_{2}$ of lysine from simplified gelatin with PEG-SG. (d) The reactivity of $-\mathrm{NH}_{2}$ of arginine from simplified gelatin with PEG-SG. The simulation was carried out at $298.15 \mathrm{~K}$ and $310.15 \mathrm{~K}$, respectively. Gelatin has been simplified as the sequence of lysine and arginine. 


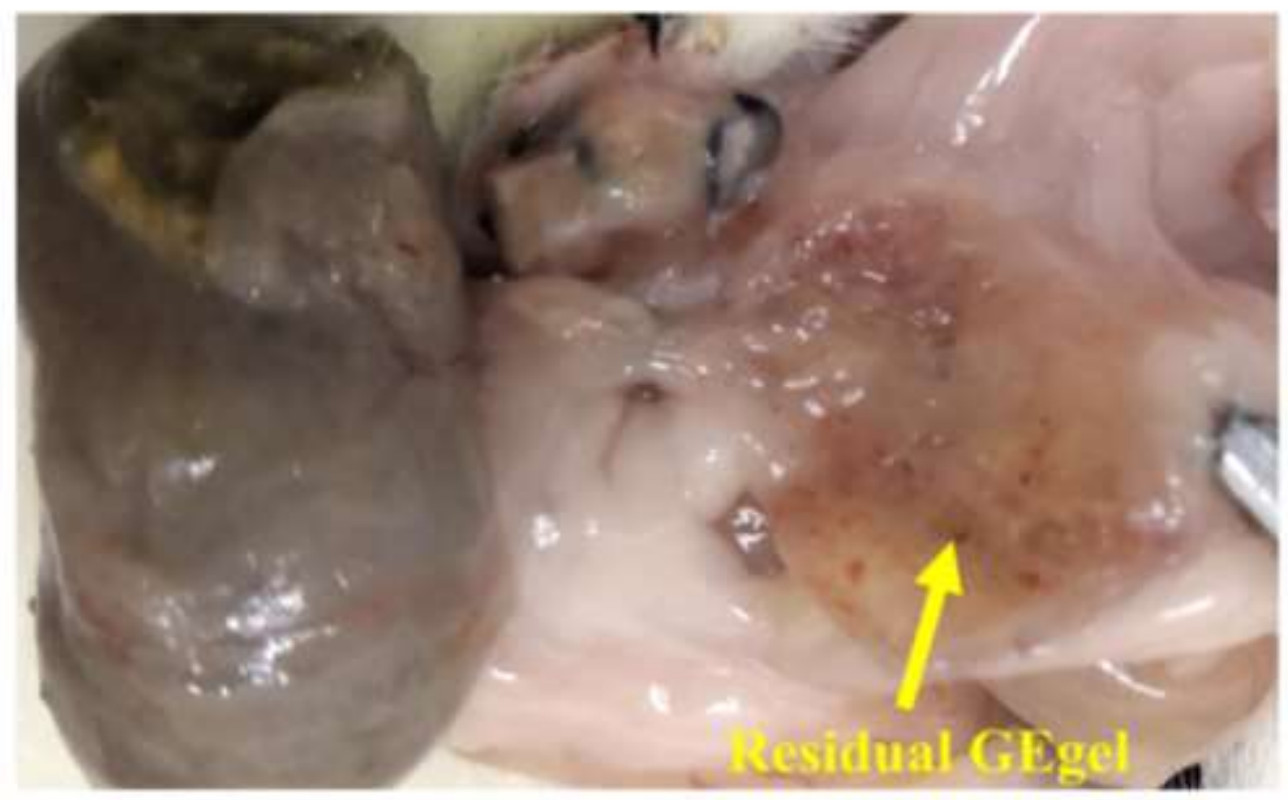

Figure S14. Residual GEgle on cecum surface after 7 days

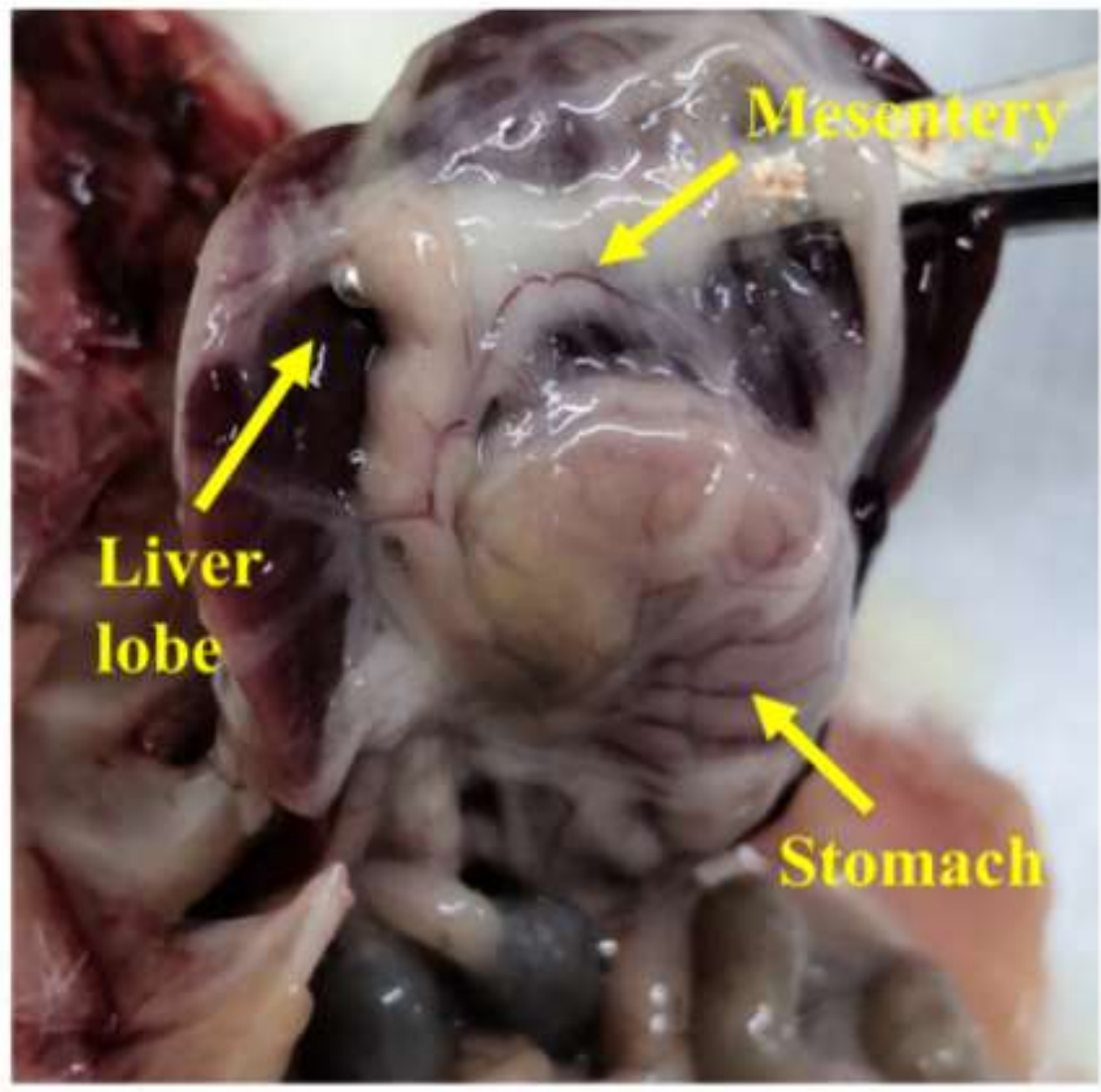

Figure S15. Severe adhesion among liver lobe, stomach and mesentery in GEgel 
treated groups 